\title{
Transdermal delivery of analgesics
}

“...understanding the composition and function of the skin has allowed effective drug administration in some circumstances."

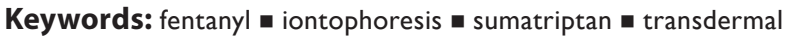

Transdermal drug delivery is possible with some drugs, depending upon their physiochemical properties. Improved convenience and efficiency for both patients and caregivers are wellrecognized advantages when compared with alternative routes of administration in selected circumstances. Penetration can be enhanced by altering the agent properties, the addition of carrier vehicles and the application of a small imperceptible electrical current, termed iontophoresis. Iontophoresis speeds the onset of therapeutic levels and thus makes transdermal application suitable for acute pain and episodic pain applications. Advances in technology could permit increased flexibility and programmability of these devices, which, in turn, might lead to expanded analgesic applications.

Transdermal application of analgesic agents represents a novel approach to drug delivery that is finding increasing clinical utility. In addition to the convenience of transdermal administration, several potential advantages in selected circumstances contribute to this trend. Specifically, oral delivery of analgesic agents, the most common route of administration, at times can present significant challenges. First, drugs having low oral bioavailability or drugs undergoing extensive first-pass metabolism, often require significantly more drug than when given parenterally. This increased dose requirement is particularly problematic when the analgesic is associated with local gastrointestinal or hepatic adverse effects: the increased drug load presents a direct gastrointestinal or hepatic burden. Moreover, pharmacogenetic variability in the hepatic metabolism of the agents can further complicate and exacerbate difficulties associated with the increased dose.

While transmucosal or, less commonly, inhaled drug delivery can bypass some of the previously mentioned challenges, these approaches are not possible with all agents. More commonly, parenteral analgesic delivery is provided by injection via subcutaneous, intramuscular, intravenous, epidural or intrathecal routes. The requirement for physical puncture of the skin or implantation of a device limits the availability and practicality in some settings.

As with the transmucosal route, transdermal drug delivery can also provide a non-invasive approach to avoiding first-pass metabolism. While local anesthetics have been successfully applied to anesthetize the skin [1] for both acute and chronic pain conditions [2], other analgesics can be delivered transdermally with the intention of penetrating to deeper structures beneath the skin and for systemic drug delivery.

\section{Skin as a barrier}

The skin, as arguably the body's most important physical barrier to intrusion from environmental toxins and other chemicals, seems an unlikely route of efficient drug administration. However, understanding the composition and function of the skin has allowed effective drug administration in some circumstances.

The efficiency of the skin as a barrier is due to both physical and functional properties. The main physical barrier is the stratum corneum. This outer layer of the epidermis consists of a blanket of flattened anucleated cells. These cells or corneocytes, are filled with keratin filaments and surrounded by crosslinked cornified envelope proteins. Nonpolar lipids engulf these cells forming a 'hydrophobic matrix'. These lipids are predominantly of three types: free fatty acids, ceramides and cholesterol. Ceramides are amide-linked fatty acids derived locally from the hydrolysis of glycosphingolipids. Similarly, most epidermal cholesterol is synthesized locally [3].

Beneath the corneal layer lie the nucleated cell layers of the epidermis. Cell-cell interconnections and an interconnecting web of proteins derived from the keratinocytes provide additional barriers to drug penetration. Tight junction proteins, adherens junctions,

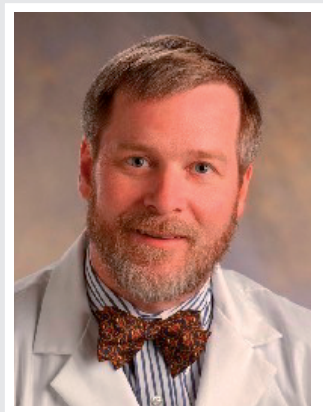

\section{Craig T Hartrick}

Oakland University William Beaumont School of Medicine, 525 O' Dowd Hall, Rochester, MI 48309, USA Tel.: + I 2489643440

Fax: + I 2489643 II2

E-mail: chartrick@beaumont.edu 
desmosomes and gap junctions formed by connexin proteins all play important roles in the maintenance of the barrier function of skin. Modulation of the barrier function following injury is mediated by local cytokine, cAMP and, importantly, calcium concentrations [3].

Topical drug application must then overcome these sequential barriers to penetration to gain access to the underlying systemic circulation in the subcutaneous tissues. The lipids in the extracellular matrix of the stratum corneum provide a contiguous pathway for diffusion, making the lipid solubility of the topically applied agent a key determinant of penetration. However, once through the first phase of the journey, the agent must then become hydrophilic for uptake into the systemic circulation. Thus, the ideal agent for passive transdermal delivery should have sufficient lipid-phase and aqueous-phase solubility to complete the transitions from skin surface to systemic absorption from the subcutaneous tissues. Moreover, the gel-like nature of the lipid matrix slows diffusion of larger molecules making molecular size a limiting factor in drug mobility through the stratum corneum [4].

Enhanced drug penetration of the skin has been associated with the use of a variety of vehicles including various fatty acids, alcohols and other permeation enhancers [5,6]. More recently, a number of novel formulations have been advanced to further improve transdermal drug delivery. These approaches have included encapsulation of the drug within a lipid vesicle, either alone or with the addition of surfactants to supposedly improve deformability, allowing larger moieties to pass through the narrow passageways within the lipid matrix, nanosuspensions of hydrophobic drugs and microemulsions [7].

\section{Localized analgesia}

Nonsteroidal anti-inflammatory drugs (NSAIDs) are potent inhibitors of cyclo-oxygenases. Consequently, they owe their analgesic properties to both peripheral (cyclooxygenase [COX]-1) and central (COX-2) inhibition of prostaglandin production. As such they have the potential to reduce both primary hyperalgesia at the site of injury in association with sensitization of the primary afferent nociceptor and, when systemic levels are sufficient to reach the CNS, secondary hyperalgesia developing centrally following activation of constitutive COX-2 in the dorsal horn [8]. However, systemic administration of NSAIDs are associated with adverse gastrointestinal events including peptic ulcers with bleeding - the selective COX-2 agents have a greatly reduced incidence of adverse events compared with agents with mixed COX-1 and COX-2 activity [9]. COX-2 agents, however, are not devoid of significant systemic effects [10]. Consequently, the ability to deliver NSAIDs directly to a site of injury without incurring significant systemic levels is appealing.

Topically administered NSAIDs can achieve effective analgesia, localized to painful tissues deep to the area of application, with reduced blood levels and, therefore, reduced adverse effects compared with systemic drug delivery [11]. Aspirin, ketoprofen, diclofenac, indomethacin and others have been used. Modification of the vehicle carrier, such as increasing the ethanol content in a gel drug-delivery formulation, can greatly enhance percutaneous permeation [12].

A number of other analgesics have been applied topically, often in combinations that include NSAIDs. Neuropathic pain formulations commonly include agents such as $\alpha 2$-adrenergics (e.g., clonidine), neurokinin-1 antagonists (e.g., capsaicin), tricyclic antidepressants (e.g., amitriptyline and doxepin), N-methyl-Daspartate antagonists (e.g., ketamine and dextromethorphan) and/or local anesthetics [13]. The simultaneous administration of agents acting on a different molecular target allows for beneficial additive analgesic effects and reduced dose of any particular agent. Consequently the systemic levels, already low following transdermal delivery, can be further reduced.

\section{Systemic analgesia}

Transdermal delivery can also be used for systemic analgesic administration. Fentanyl and buprenorphine are available in transdermal patches [14]. Relatively consistent drug release occurs when the patch is on, which maintains systemic levels of the drug, with some short-term residual reservoir effect in the subcutaneous tissues after patch removal. However, the peak systemic concentration is not reached for hours after application. Consequently, these opioid patches are not typically used in acute pain situations; they are more often used to treat chronic or cancer pain.

To effectively treat acute or episodic pain a rapid onset of effect is desirable. Control over rapidly changing pain levels is facilitated by repeated administration of a fast-acting analgesic having short duration, simulating intravenous patient-controlled analgesia (PCA) bolus administration. Rapid penetration of a drug through 
the skin can be facilitated by iontophoresis. While this approach has been used for decades to facilitate delivery of NSAIDs, local anesthetics and other analgesics, new devices have recently been developed to allow patients themselves to safely control the delivery of their medication.

Using iontophoresis, a battery provides sufficient current density to the skin to force charged molecules into the skin. While the molecular size and charge directly affect repulsion from the driving electrode [15], the electric current can also alter the permeability of the skin and electroosmosis may even alter transport of uncharged molecules [5]. Iontophoretic transdermal fentanyl was initially US FDA approved for acute pain, but is not yet marketed [16]. As initially developed, this small $(7 \times 4.5 \mathrm{~cm})$, low-profile $(3 \mathrm{~mm}$ in height) device adheres directly to the skin. It consists of two parts housed within a single case: electronics and a drug reservoir. It allows patients to rapidly deliver a fixed aliquot of medication on demand by pressing a button on the surface of the device to activate the current, but has a 10 -min lock-out period to reduce stacking of doses, thus providing a safe and efficient drug-delivery system for acute pain [17].

Recently, rapid delivery of sumatriptan for the treatment of episodic migraine headaches has been successfully administered by iontophoresis [18]. While other antimigraine agents have been delivered by iontophoresis in studies, the sumatriptan system has been submitted for FDA approval. The transdermal route is particularly appropriate for this condition given the high incidence of nausea associated with acute attacks in migraineurs, making oral delivery of drugs problematic. Moreover, transdermal delivery bypasses the first-pass effect and avoids gastrointestinal absorption, which can also be delayed. Iontophoresis allows more rapid attainment of therapeutic levels compared with oral administration without the sudden increase in levels seen following subcutaneous injection of sumatriptan. This blunted rate of rise in systemic levels may reduce some of the adverse effects commonly seen with serotonin antagonists, such as chest discomfort and anxiety [18].

\section{Future perspective}

Monitoring compliance with therapy in outpatient treatment and drug accountability, especially for inpatient treatment, are issues that are currently receiving considerable attention. This is particularly relevant when administering opioids. The current version of the transdermal iontophoretic fentanyl system can only approximate the number of doses delivered (using a flashing light) and cannot be queried regarding when these doses were delivered. The ability to determine the precise number and timing of patient attempts and delivered doses from a transdermal iontophoretic system, just as one typically can from a standard PCA pump, is desirable. Future versions of these types of devices might contain electronics capable of providing digital read-outs displaying detailed drug-delivery attempts and timing information. Wireless technology might also provide the ability to transmit this data directly to an electronic medical record.

\section{“...wireless technology could potentially allow remote programming of transdermal iontophoretic devices to provide increased delivery aliquots, altered lock-out intervals or even continuous basal delivery."}

Once these developments take place, the principal use for traditional intravenous PCA pumps might be reserved for administering drugs that are not suitable for transdermal delivery, or when dose adjustment is needed. While one of the obvious advantages of a fixed-dose system is the elimination of programming errors, patients that are opioid tolerant may not benefit from typical standard therapeutic dosing. Patients requiring the flexibility of titration of doses or the addition of a basal infusion would still require traditional PCA devices. However, in the future, wireless technology could potentially allow remote programming of transdermal iontophoretic devices to provide increased delivery aliquots, altered lock-out intervals or even continuous basal delivery. Programmability of these small devices might eventually largely supplant the need for bulky pumps and intravenous access. Custom settings for home use, as in the case of sumatriptan for migaineurs, might also be helpful in some situations.

Financial \& competing interests disclosure Craig $T$ Hartrick has received research grant funding from Ortho-McNeil and has been a consultant for both Ortho$\mathrm{McNeil}$ and Incline, developers of transdermal iontophoretic fentanyl delivery systems. The author has no other relevant affiliations or financial involvement with any organization or entity with a financial interest in or financial conflict with the subject matter or materials discussed in the manuscript apart from those disclosed.

No writing assistance was utilized in the production of this manuscript. 


\section{Bibliography}

1 Ehrenstrom Reiz GM, Reiz SL. EMLA - a eutetic mixture of local anaesthetics for topical anaesthesia. Acta Anaesthesiol. Scand. 26(6), 596-598 (1982).

2 Gammaitoni AR, Alvarez NA, Galer BS. Safety and tolerability of the lidocaine patch $5 \%$, a targeted peripheral analgesic: a review of the literature. J. Clin. Pharmacol. 43(2), 111-117 (2003).

3 Proksch E, Brandner JM, Jensen J-M. The skin: an indispensible barrier. Exp. Dermatol. 17, 1063-1072 (2008).

4 Naik A, Kalia YN, Guy RH. Transdermal drug delivery: overcoming the skin's barrier function. Pharm. Sci. Technol. Today 3(9), 318-326 (2000).

5 Barry BW. Novel mechanisms and devices to enable successful transdermal drug delivery. Eur. J. Pharm. Sci. 14, 101-114 (2001).

6 Mittal A, Sara UV, Ali A, Aqil M. Status of fatty acids as skin penetration enhancers - a review. Curr. Drug Deliv. 6(3), 274-279 (2009).

7 Dhamankar AK, Manwar JV, Kumbhar DD. The novel formulation design of $\mathrm{O} / \mathrm{W}$ microemulsion of ketoprofen for improving transdermal absorption. Int. J. Pharm. Tech. Res. 1(4), 1449-1457 (2009).
8 Hartrick CT. Multimodal postoperative pain management. Am. J. Health Syst. Pharmacy 61(Suppl. 1), S4-S10 (2004).

9 Hawkey C, Laine L, Simon T et al. Comparison of the effect of rofecoxib (a cyclooxygenase 2 inhibitor), ibuprofen, and placebo on the gastrointestinal mucosa of patients with osteoarthritis: a randomized, double-blind, placebo-controlled trial. Arthritis Rheum. 43(2), 370-377 (2000).

10 Kerr SJ, Sayer GP, Whicker SD, Rowett DS, Saltman DC, Mant A. All-cause mortality of elderly Australian veterans using COX-2 selective or non-selective NSAIDs: a longitudinal study. Br. J. Clin. Pharmacol. DOI: $10.1111 /$ j.1365-2123.2010.03702.x (2010) (Epub ahead of print).

11 Tugwell PS, Wells GA, Shainhouse JZ. Equivalence study of topical diclofenac solution (pennsaid) compared with oral diclofenac in symptomatic treatment of osteoarthritis of the knee: a randomized controlled trial. J. Rheumatol. 31(10), 2002-2012 (2004).

12 El-Kattan AF, Asbill CS, Kim N, Micjniak BB. Effect of formulation variables on percutaneous permeation of ketoprofen from gel formulations. Drug Deliv. 7(3), 147-153 (2000).
13 de Leon-Casasola OA. Multimodal approaches to the management of neuropathic pain: the role of topical analgesia. J. Pain Symptom Mange. 33(3), 356-364 (2007).

14 Freye E, Anderson-Hillemacher A, Ritzdorf I, Levy JV. Opioid rotation from high-dose morphine to transdermal buprenorphine (Transtec $\left.^{\circledR}\right)$ in chronic pain patients. Pain Pract. 7(2), 123-129 (2007).

15 Kalia YN, Naik A, Garrison J, Guy RH. Iontophoretic drug delivery. Adv. Drug Del. Rev. 56, 619-658 (2004).

16 Hartrick CT. Iontophoretic transdermal fentanyl. In: The Essence of Analgesia and Analgesics. Sinatra RS, Jahr JS, WatkinsPitchford JM (Eds). Cambridge University Press, NY, USA, 455-458 (2011).

17 Hartrick CT. Patient-controlled transdermal iontophoretic fentanyl system as an alternative to intravenous morphine PCA. Future Neurology 2(6), 621-627 (2007).

18 Rapoport AM, Freitag F, Perlman SH. Innovative delivery systems for migraine: the clinical utility of a transdermal patch for acute treatment of migraine. CNS Drugs 24(11), 929-940 (2010). 